Pacific

Journal of

Mathematics

\title{
DISJOINTIFICATION INEQUALITIES IN SYMMETRIC QUASI-BANACH SPACES AND THEIR APPLICATIONS
}

Sergey Astashkin, Fedor A. Sukochev and Dmitriy Zanin 


\title{
DISJOINTIFICATION INEQUALITIES IN SYMMETRIC QUASI-BANACH SPACES AND THEIR APPLICATIONS
}

\author{
Sergey Astashkin, Fedor A. Sukochev And DMitriy Zanin
}

We demonstrate the relevance of the Prokhorov inequality to the study of Khintchine-type inequalities in symmetric function spaces. Our main result shows that the latter inequalities hold for a pair of quasi-Banach symmetric function spaces $X$ and $Y$ if and only if the Kruglov operator $K$ acts from $X$ to $Y$. We also obtain an extension of von Bahr-Esseen and Esseen-Janson $L_{p}$-estimates for sums of independent mean zero random variables to the class of quasi-Banach symmetric spaces. In particular, in contrast to the well-known Esseen-Janson theorem, we do not assume that the summands are equidistributed.

\section{Introduction}

The classical Khintchine inequality [1923] describes the span of independent centered $\{ \pm 1\}$-valued Bernoulli random variables in quasi-Banach $L_{p}$-spaces. A particular case of the latter sequence is given by the Rademacher functions $r_{n}(t):=\operatorname{sgn} \sin \left(2^{n} \pi t\right), t \in[0,1), n \geq 1$. In this case, for all $p \in(0, \infty)$ the sequence $\left\{r_{n}\right\}_{n=1}^{\infty}$ in the $L_{p}$-spaces on the interval $(0,1)$ (equipped with Lebesgue measure $m$ ) is equivalent to the unit vector basis $\left\{e_{n}\right\}_{n=1}^{\infty}$ of $l_{2}$. A famous extension of this inequality to a more general case of random variables was given later by Marcinkiewicz and Zygmund (see [1937, Theorem 13, p. 87] and [1938, Theorem 5, p. 109]): for every $1 \leq p<\infty$ there are constants $A_{p}>0$ and $B_{p}>0$ such that for any $n \in \mathbb{N}$ and for an arbitrary sequence of independent mean zero random variables $\left(f_{k}\right)_{k \in \mathbb{N}}$ from $L_{p}(0,1)$ we have

$$
A_{p}\left\|\left(\sum_{k=1}^{n} f_{k}^{2}\right)^{1 / 2}\right\|_{p} \leq\left\|\sum_{k=1}^{n} f_{k}\right\|_{p} \leq B_{p}\left\|\left(\sum_{k=1}^{n} f_{k}^{2}\right)^{1 / 2}\right\|_{p} .
$$

In the special setting of Banach symmetric function spaces Johnson and Schechtman [1988] proved a far reaching generalization of the Marcinkiewicz-Zygmund inequality (1). More precisely, they established that if such a space $X$ is either separable or has the Fatou property (for the relevant definitions see the following

MSC2010: primary 46E30; secondary 60G50, 46B09.

Keywords: Kruglov operator, Prokhorov inequality, quasi-Banach spaces. 
section) and the lower Boyd index of $X$ is strictly positive, then (1) holds (even for a more general case of martingale differences). Later on, Astashkin [2008] showed that inequality (1) holds in a Banach symmetric space $X$ if and only if $X$ satisfies the so-called Kruglov property. The latter property, introduced by Braverman [1994], has its origin in a remarkable result due to Rosenthal [1970] that for sequences $\left\{f_{n}\right\}_{n=1}^{\infty}$ of independent mean zero random variables in $L_{p}(0,1)$, $p \geq 2$, the mapping $f_{n} \rightarrow f_{n}(t-n+1) \chi_{[n-1, n)}(t), t \in \mathbb{R}$, extends to an isomorphism between the closed linear span $\left[f_{n}\right]_{n=1}^{\infty}\left(\right.$ taken in $\left.L_{p}(0,1)\right)$ and the closed linear span $\left[f_{n}(t-n+1) \chi_{[n-1, n)}\right]_{n=1}^{\infty}$ (taken in $\left.L_{p}(0, \infty) \cap L_{2}(0, \infty)\right)$. The main focus of the present paper is to establish optimal conditions on a quasinormed symmetric function space in which inequalities of the type (1) hold. Our techniques are centered around the so-called Kruglov operator, a natural generalization of the Kruglov property, which was introduced in [Astashkin and Sukochev 2005] (see also [Astashkin and Sukochev 2010]). The usage of this operator allows us to make a straightforward connection between sums of independent random variables and their disjoint translates. Another major ingredient of our approach consists in utilizing Prokhorov's famous inequality [1959] (see also Theorem 17 below) which allows us to treat the problem in the full generality.

Using our present method, we also provide a far-reaching extension of the wellknown von Bahr-Esseen and Esseen-Janson $L_{p}$-estimates for sums of independent mean zero random variables (see [von Bahr and Esseen 1965] and [Esseen and Janson 1985]). We extend inequalities of such type to the class of quasi-Banach symmetric spaces, and, at the same time, we do not assume that the summands are equally distributed (which is in strong contrast with Esseen and Janson's approach [1985, Theorem 4]). Note that earlier, Braverman [1994, § II.2] generalized the von Bahr-Esseen inequality to (Banach) symmetric spaces with the Kruglov property.

\section{Preliminaries}

2.1. Quasi-Banach spaces. Let $X$ be a linear space over the field of real numbers $\mathbb{R}$. A function $\|\cdot\|_{X}: X \rightarrow \mathbb{R}$ is called a quasinorm if the following conditions hold:

(a) $\|x+y\|_{X} \leq C\left(\|x\|_{X}+\|y\|_{X}\right)$ for every $x, y \in X$ and some constant $C>0$.

(b) $\|c x\|_{X}=|c| \cdot\|x\|_{X}$ for every $x \in X$ and $c \in \mathbb{R}$.

(c) $\|x\|_{X} \geq 0$. Moreover, $\|x\|_{X}=0$ if and only if $x=0$.

The least of all constants $C$ satisfying condition (a) above is called the modulus of concavity of the quasinorm $\|\cdot\|_{X}$ and is denoted by $C(X)$.

If $X$ is a linear space over $\mathbb{R}$ and if $\|\cdot\|_{X}: X \rightarrow \mathbb{R}$ is a quasinorm, then $X=\left(X,\|\cdot\|_{X}\right)$ is called a quasinormed space. If every Cauchy sequence in a quasinormed space $X$ converges, then $X$ is called a quasi-Banach space. 
For example, $L_{p}(0,1)$ and $L_{p}(0, \infty), 0<p<1$, are quasi-Banach spaces with modulus of concavity $C(p)=C\left(L_{p}\right)=2^{1 / p-1}$.

Recall that a quasinorm $\|\cdot\|_{X}$ in $X$ is said to be a $p$-norm, $0<p<1$, if for any $x_{1}, x_{2} \in X$ we have

$$
\left\|x_{1}+x_{2}\right\|_{X}^{p} \leq\left\|x_{1}\right\|_{X}^{p}+\left\|x_{2}\right\|_{X}^{p} .
$$

By the Aoki-Rolewicz theorem [Kalton et al. 1984], for any quasinorm $\|\cdot\|_{X}$ there exists $0<p<1$ such that $\|\cdot\|_{X}$ is a $p$-norm.

2.2. Symmetric function spaces. We are interested in those quasi-Banach spaces which consist of Lebesgue-measurable functions either on $(0,1)$ or on $(0, \infty)$.

For a Lebesgue-measurable, a.e. finite function $x$ on $(0,1)$ (or $(0, \infty))$ we define its distribution function by

$$
d_{x}(s):=m(\{t: x(t)>s\}), \quad s \in \mathbb{R},
$$

where $m$ stands for Lebesgue measure. Let $S(0,1)$ (respectively, $S(0, \infty)$ ) denote the space of all Lebesgue-measurable functions $x$ on $(0,1)$ (respectively, on $(0, \infty)$ with $d_{|x|}(s)<\infty$ for sufficiently large $s$ ).

Two measurable functions $x$ and $y$ are called equimeasurable (written $x \sim y$ ) if their distribution functions $d_{x}$ and $d_{y}$ coincide. In particular, for every measurable function $x$, the function $|x|$ is equimeasurable with its decreasing rearrangement $x^{*}$, defined by the formula

$$
x^{*}(t):=\inf \left\{\tau \geq 0: d_{|x|}(\tau)<t\right\}, \quad t>0 .
$$

If $x, y \geq 0$, then $x^{*}=y^{*}$ if and only if $x$ and $y$ are equimeasurable. We recall that a function $x$ is said to be symmetrically distributed if $x$ and $-x$ are equimeasurable.

As it is traditional in probability theory, we denote by $\phi_{x}$ the characteristic function of an element $x \in S(0,1)$; that is, $\phi_{x}(t)=\int_{0}^{1} e^{i t x(s)} d s$. Recall that functions $x, y \in S(0,1)$ are equimeasurable if and only if their characteristic functions $\phi_{x}$ and $\phi_{y}$ coincide.

Definition 1. Let $X \subset S(0,1)$ (or $X \subset S(0, \infty)$ ) be a quasi-Banach space.

(a) $X$ is said to be a quasi-Banach function space if, from $x \in X, y \in S(0,1)$ (or $y \in S(0, \infty))$ and $|y| \leq|x|$, it follows that $y \in X$ and $\|y\|_{X} \leq\|x\|_{X}$.

(b) A quasi-Banach function space $X$ is said to be symmetric if, for every $x \in X$ and any measurable function $y$, the assumption $y^{*}=x^{*}$ implies that $y \in X$ and $\|y\|_{X}=\|x\|_{X}$.

Without loss of generality, in what follows we assume that $\left\|\chi \chi_{(0,1)}\right\|_{X}=1$, where $\chi_{E}$ denotes the indicator function of a Lebesgue measurable set $E$. 
The following assertion is well known in the Banach-space setting (see, for instance, [Lindenstrauss and Tzafriri 1979, Proposition 1.d.2]). For the reader's convenience, we provide a short proof.

Lemma 2. Let $X$ be a quasi-Banach function space. If $0 \leq x$ and $y \in X$, then $\left\|(x y)^{1 / 2}\right\|_{X} \leq C(X)\|x\|_{X}^{1 / 2}\|y\|_{X}^{1 / 2}$.

Proof. It is easy to see that

$$
(x y)^{1 / 2} \leq \frac{1}{2}\left(\theta x+\theta^{-1} y\right), \quad \theta>0,
$$

and, therefore,

$$
\left\|(x y)^{1 / 2}\right\|_{X} \leq \frac{C(X)}{2}\left(\theta\|x\|_{X}+\theta^{-1}\|y\|_{X}\right) .
$$

Taking the infimum over all $\theta>0$, we infer

$$
\left\|(x y)^{1 / 2}\right\|_{X} \leq C(X)\|x\|_{X}^{1 / 2}\|y\|_{X}^{1 / 2} .
$$

Let $X$ be a quasi-Banach symmetric function space and let $x_{n} \in X, n \in \mathbb{N}$, be such that $\sup _{n \in \mathbb{N}}\left\|x_{n}\right\|_{X}<\infty$ and $x_{n} \rightarrow x$ almost everywhere. If, for every such sequence, we have $x \in X$ and $\|x\|_{X} \leq \liminf _{n \rightarrow \infty}\left\|x_{n}\right\|_{X}$, then $X$ is said to satisfy the Fatou property.

Suppose that $X$ is a separable quasi-Banach symmetric space on $(0,1)$. Denote by $\bar{X}$ the set of all $x \in S(0,1)$ such that $\lim _{a \rightarrow+\infty}\left\|[|x|]_{a}\right\|_{X}<\infty$, where $[|x|]_{a}:=|x|$ if $|x|<a$ and $[|x|]_{a}:=0$ if $|x| \geq a$. The set $\bar{X}$, equipped with the norm $\|x\|_{\bar{X}}:=$ $\lim _{a \rightarrow+\infty}\left\|[|x|]_{a}\right\|_{X}$, becomes a quasi-Banach symmetric space with the Fatou property. Moreover, $X$ embeds isometrically into $\bar{X}$. It can be easily checked that for every quasi-Banach symmetric space $X$ on $(0,1)$ the continuous embedding $X \supset L_{\infty}(0,1)$ holds. Then, the closure of $L_{\infty}(0,1)$ in $X$, denoted by $X_{0}$, is a separable quasi-Banach symmetric space with the norm $\|\cdot\|_{X}$ whenever $X \neq$ $L_{\infty}(0,1)$.

If $\tau>0$, the dilation operator $\sigma_{\tau}$ is defined by setting $\sigma_{\tau} x(s)=x(s / \tau), s>0$, in the case of the semiaxis. In the case of the interval $(0,1)$, the operator $\sigma_{\tau}$ is defined by

$$
\sigma_{\tau} x(s):= \begin{cases}x(s / \tau) & \text { if } s \leq \min \{1, \tau\} \\ 0 & \text { if } \tau<s \leq 1 .\end{cases}
$$

Below we shall often consider the probability product space

$$
(\Omega, \mathbb{P}):=\prod_{k=0}^{\infty}\left((0,1), m_{k}\right),
$$

( $m_{k}$ is the Lebesgue measure on $\left.(0,1), k \geq 0\right)$. Observe that in an arbitrary symmetric space the norms of any two elements with identical distribution coincide. 
Hence, using a one-to-one measure-preserving transformation between measure spaces $(\Omega, \mathbb{P})$ and $((0,1), m)$, we will identify an arbitrary measurable function $x(\omega)=x\left(\omega_{0}, \omega_{1}, \ldots, \omega_{n}, \ldots\right)$ on $(\Omega, \mathbb{P})$ with the corresponding element from $S(0,1)$. Since a particular form of the measure-preserving transformation used in such identification is not important, we completely suppress it from the notations. Thus, we will view the set $\Omega$ as $(0,1)$ and any measurable function on $(\Omega, \mathbb{P})$ as a function from $S(0,1)$ and vice versa. A reader interested in more details of such identification is referred to [Astashkin and Sukochev 2010].

Let $x_{k}, k \geq 0$, be elements from $S(0,1)$ and let $y_{k} \in S(0, \infty), k \geq 0$, be their disjoint copies; that is, $x_{k} \sim y_{k}$ for all $k \geq 0$, and $y_{l} y_{m}=0$ if $l \neq m$. For the function $\sum_{k \geq 0} y_{k}$, which is frequently called the disjoint sum of $x_{k}, k \geq 0$, we shall use the suggestive notation $\bigoplus_{k \geq 0} x_{k}$. It is important to observe that the distribution function of a disjoint sum $\bigoplus_{k \geq 0} x_{k}$ does not depend on the particular choice of elements $y_{k}, k \geq 0$. In the special case when $\sum_{k=1}^{n} m\left(\operatorname{supp}\left(x_{k}\right)\right) \leq 1$, $n \in \mathbb{N}$, it is convenient to view the sum $\bigoplus_{k \geq 0} x_{k}$ as a measurable function on $(0,1)$.

The following useful construction was introduced in [Johnson et al. 1979] (see also [Lindenstrauss and Tzafriri 1979, 2.f]). If $X$ is a quasi-Banach symmetric function space on $(0,1)$ and $0<p \leq \infty$, then the set $Z_{X}^{p}$ consists of all $f \in S(0, \infty)$ such that

$$
\|f\|_{Z_{X}^{p}}:=\left\|f^{*} \chi_{(0,1)}\right\|_{X}+\left\|\min \left\{f^{*}, f^{*}(1)\right\}\right\|_{p}<\infty .
$$

It can be easily checked that the functional $\|\cdot\|_{Z_{X}^{p}}$ is a quasinorm on $Z_{X}^{p}$.

2.3. Kruglov operator and Kruglov property. The Kruglov property was introduced by Braverman [1994] when he compared sums of independent functions with sums of their disjoint copies in (Banach) symmetric spaces. Such terminology stems from related probabilistic constructions, due to Kruglov [1970], used in the study of infinitely divisible distributions (e.g., in analysis of the classical Levy-Khintchine formula).

Let $x \in S(0,1)$. By $\pi(x)$ we denote the random variable $\sum_{i=1}^{N} x_{i}$, where $x_{i}$, $i=1, \ldots, N$, are independent copies of $x$ and $N$ is a random variable having Poisson distribution with parameter 1 and independent with respect to the sequence $\left\{x_{i}\right\}$.

Definition 3. A quasi-Banach symmetric space $X$ on $(0,1)$ is said to have the Kruglov property $(X \in \mathbb{K})$ if from $x \in X$ it follows that $\pi(x) \in X$.

Simplifying the situation, the Kruglov property holds for spaces sufficiently "remote" from the space $L_{\infty}(0,1)$. For example, if a symmetric Banach function space $X$ contains $L_{p}(0,1)$ for some $p<\infty$, then $X$ possesses the Kruglov property (see, e.g., [Braverman 1994, Theorem 1.2] or [Astashkin and Sukochev 2010]). For 
a more precise characterization of various classes of (Banach) symmetric function spaces possessing the Kruglov property, we refer the reader to [Astashkin and Sukochev 2005; 2007; 2010; Braverman 1994].

Now, we recall the definition of the Kruglov operator, which can be viewed as a natural generalization of the notion of the Kruglov property. Let $\left\{B_{n}\right\}_{n=0}^{\infty}$ be a fixed sequence of mutually disjoint measurable subsets of $(0,1)$ such that $m\left(B_{n}\right)=1 /(e n !)$. Define the operator $K: S(0,1) \rightarrow S(0,1)$ by setting

$$
K x(\omega):=\sum_{n=1}^{\infty} \sum_{k=1}^{n} x\left(\omega_{k}\right) \chi_{B_{n}}\left(\omega_{0}\right) .
$$

It is not difficult to see that

$$
\phi_{K x}(t)=\phi_{\pi(x)}(t)=\exp \left(\phi_{x}(t)-1\right), \quad t \in \mathbb{R} .
$$

Therefore, by the definition of the Kruglov property, a quasi-Banach symmetric function space $X$ has the Kruglov property if and only if the operator $K$ acts boundedly in $X$. Though the following crucial theorem originated in [Astashkin and Sukochev 2005], the first explicit statement (with a proof) appeared in [Astashkin et al. 2011].

Theorem 4. If a sequence $\left\{x_{k}\right\}_{k=1}^{n} \subset S(0,1), n \in \mathbb{N}$, consists of disjointly supported functions, then the sequence $\left\{K x_{k}\right\}_{k=1}^{n}$ consists of independent functions.

We will need also the following assertion, which is an immediate consequence of [Astashkin and Sukochev 2010, Theorem 15].

Theorem 5. If $X$ is a separable quasi-Banach symmetric space on $(0,1)$ such that $K: \bar{X} \rightarrow \bar{X}$, then $K: X \rightarrow X$ and $\|K\|_{X \rightarrow X}=\|K\|_{\bar{X} \rightarrow \bar{X}}$.

\section{Disjointification inequalities for positive functions}

We will use the following approximation to the function $K x$, where $x$ is an arbitrary measurable function on the interval $(0,1)$. For every $n \in \mathbb{N}$ define the operator $H_{n}: S(0,1) \rightarrow S(0,1)$ by the formula

$$
H_{n} x(\omega):=\sum_{k=1}^{n}\left(\sigma_{1 / n} x\right)\left(\omega_{k}\right) .
$$

The following result is well known (see the proof of Lemma 1.6 in [Braverman 1994] or of Theorem 22 in [Astashkin and Sukochev 2010]). However, we present its proof for the reader's convenience.

Lemma 6. The sequence of functions $\left\{H_{n} x\right\}_{n=1}^{\infty}$ converges to the function $K x$ in distribution. 
Proof. It is not difficult to see that $\phi_{H_{n} x}=\phi_{\sigma_{1 / n} x}^{n}$. On the other hand,

$$
\phi_{\sigma_{1 / n} x}(t)=\int_{0}^{1} e^{i t \sigma_{1 / n} x(s)} d s=\left(1-\frac{1}{n}\right)+\frac{1}{n} \phi_{x}(t) .
$$

Therefore, by (2), we obtain

$$
\phi_{H_{n} x}=\left(1+\frac{\phi_{x}-1}{n}\right)^{n} \rightarrow \exp \left(\phi_{x}-1\right)=\phi_{K x} .
$$

Since the convergence of distributions follows from the convergence of characteristic functions [Borovkov 1998, Theorem 6.2.1], the result follows.

Theorem 7. Let $X$ and $Y$ be quasi-Banach symmetric spaces on $(0,1)$ and let $Y$ have the Fatou property. Suppose that there exists a positive constant $C>0$ such that for every sequence of nonnegative independent functions $\left\{x_{k}\right\}_{k=1}^{n} \subset X, n \in \mathbb{N}$, with $\sum_{k=1}^{n} m\left(\operatorname{supp}\left(x_{k}\right)\right) \leq 1$, we have

$$
\left\|\sum_{k=1}^{n} x_{k}\right\|_{Y} \leq C \cdot\left\|\bigoplus_{k=1}^{n} x_{k}\right\|_{X} .
$$

Then the operator $K$ maps $X$ into $Y$ and $\|K\|_{X \rightarrow Y} \leq C$.

The assertion remains valid under the assumption that the inequality (4) holds for $X=Y$, where $X$ is a separable quasi-Banach symmetric space.

Proof. For every $x \in X$, let us define $x_{k}(\omega)=\left(\sigma_{1 / n} x\right)\left(\omega_{k}\right), \omega \in \Omega$. It follows from the definition of disjoint sum that

$$
\bigoplus_{k=1}^{n} x_{k} \sim x \quad \text { for every } n \in \mathbb{N} \text {. }
$$

Therefore, applying (3) and (4), we obtain $\left\|H_{n} x\right\|_{F} \leq C\|x\|_{E}$. Furthermore, by Lemma 6 , the sequence $\left\{H_{n} x\right\}_{n \geq 1}$ converges to the function $K x$ in distribution when $n \rightarrow \infty$ and hence $\left(H_{n} x\right)^{*} \rightarrow(K x)^{*}$ almost everywhere on $(0,1)$. Since $Y$ has the Fatou property, it follows that $K x \in Y$ and $\|K x\|_{Y} \leq C\|x\|_{X}$.

Suppose now that $X$ is a separable quasi-Banach symmetric space such that (4) holds for every sequence of nonnegative independent functions $\left\{x_{k}\right\}_{k=1}^{n} \subset X$ such that $\sum_{k=1}^{n} m\left(\operatorname{supp}\left(x_{k}\right)\right) \leq 1, n \in \mathbb{N}$. From the definition of the space $\bar{X}$ (see Section 2), it follows that a similar inequality with the same constant $C$ holds also for every sequence of nonnegative independent functions $\left\{x_{k}\right\}_{k=1}^{n} \subset \bar{X}$ with $\sum_{k=1}^{n} m\left(\operatorname{supp}\left(x_{k}\right)\right) \leq 1, n \in \mathbb{N}$. Therefore, since $\bar{X}$ has the Fatou property, by the first part of theorem, we conclude that $K: \bar{X} \rightarrow \bar{X}$ and $\|K\|_{\bar{X} \rightarrow \bar{X}} \leq C$. An application of Theorem 5 completes the proof. 
Our next purpose is to establish the main result of this section (Theorem 16), which is in a sense converse to the assertion of the preceding theorem. The first step in its proof is Proposition 9 below. We also need some preparatory results.

Lemma 8. For every positive $x \in S(0,1)$, we have $\sigma_{1 / 2} x^{*} \leq(K x)^{*}$.

Proof. Let $B_{n}, n \geq 1$, be the sets from the definition of the Kruglov operator $K$. Since the $B_{n}$ are pairwise disjoint and

$$
\sum_{n=1}^{\infty} m\left(B_{n}\right)=\frac{e-1}{e}>\frac{1}{2}
$$

we may select a measurable set $B \subset \bigcup_{n \geq 1} B_{n}$ such that $m(B)=1 / 2$. It is clear that $(K x)(\omega) \geq x\left(\omega_{1}\right) \chi_{B}\left(\omega_{0}\right)$ for every $\omega \in \Omega$. Since the function $x\left(\omega_{1}\right) \chi_{B}\left(\omega_{0}\right)$ is equimeasurable with the function $\sigma_{1 / 2} x^{*}$, the assertion follows immediately.

Proposition 9. Suppose that the operator $K$ maps boundedly $X$ into $Y$, where $X$ and $Y$ are quasi-Banach symmetric spaces on $(0,1)$. If $\left\{x_{k}\right\}_{k=1}^{n}, n \in \mathbb{N}$, is a sequence of independent functions from $X$ and if $\sum_{k=1}^{n} m\left(\operatorname{supp}\left(x_{k}\right)\right) \leq 1$, then

$$
\left\|\sum_{k=1}^{n} x_{k}\right\|_{Y} \leq 2 C(Y)\|K\|_{X \rightarrow Y}\left\|\bigoplus_{k=1}^{n} x_{k}\right\|_{X} .
$$

Proof. Without loss of generality, it may be assumed that $x_{k} \geq 0,1 \leq k \leq n$. Let $y_{k} \in S(0,1)$ be pairwise disjoint copies of $x_{k}, 1 \leq k \leq n$. By Theorem 4 , the sequence $\left\{K y_{k}\right\}_{k=1}^{n}$ consists of independent functions. Observing that $K\left(\bigoplus_{k=1}^{n} x_{k}\right)$ is equimeasurable with $\sum_{k=1}^{n} K y_{k}$, and the latter is equimeasurable with the function $\sum_{k=1}^{n}\left(K x_{k}\right)^{*}\left(\omega_{k}\right)$, we arrive at

$$
\left\|\sum_{k=1}^{n}\left(K x_{k}\right)^{*}\left(\omega_{k}\right)\right\|_{Y}=\left\|\sum_{k=1}^{n} K y_{k}\right\|_{Y} \leq\|K\|_{X \rightarrow Y}\left\|\bigoplus_{k=1}^{n} x_{k}\right\|_{X} .
$$

By Lemma 8, we have

$$
\sum_{k=1}^{n}\left(\sigma_{1 / 2} x_{k}^{*}\right)\left(\omega_{k}\right) \leq \sum_{k=1}^{n}\left(K x_{k}\right)^{*}\left(\omega_{k}\right),
$$

and, therefore,

$$
\left\|\sum_{k=1}^{n}\left(\sigma_{1 / 2} x_{k}^{*}\right)\left(\omega_{k}\right)\right\|_{Y} \leq\|K\|_{X \rightarrow Y}\left\|\bigoplus_{k=1}^{n} x_{k}\right\|_{X} .
$$

For an arbitrary $k \in \mathbb{N}$, let $x_{k}^{(1)}$ and $x_{k}^{(2)}$ be disjointly supported elements of $S(0,1)$ equimeasurable with the function $\sigma_{1 / 2} x_{k}^{*}$. A moment's reflection shows that the sum $x_{k}^{(1)}+x_{k}^{(2)}$ is equimeasurable with the function $x_{k}^{*}, k \in \mathbb{N}$. Hence, the 
function $\sum_{k=1}^{n} x_{k}$ is equimeasurable with the sum $y_{0}+y_{1}$, where

$$
y_{i}(\omega):=\sum_{k=1}^{n} x_{k}^{(i)}\left(\omega_{k}\right), \quad i=0,1,
$$

which immediately implies

$$
\left\|\sum_{k=1}^{n} x_{k}\right\|_{Y}=\left\|y_{0}+y_{1}\right\|_{Y} \leq C(Y)\left(\left\|y_{0}\right\|_{Y}+\left\|y_{1}\right\|_{Y}\right) \leq 2 C(Y)\left\|\sum_{k=1}^{n} \sigma_{1 / 2} x_{k}^{*}\left(\omega_{k}\right)\right\|_{Y} \text {. }
$$

The assertion follows now from inequality (5).

Our next objective is to omit the assumption $\sum_{k=1}^{n} m\left(\operatorname{supp}\left(x_{k}\right)\right) \leq 1$. The main step is a disjointification inequality for bounded functions obtained below in Proposition 14. Let us start with some technical lemmas.

Lemma 10. Let

$$
s_{k}:=\sum_{n=k}^{\infty} \frac{1}{e \cdot n !}, \quad k \in \mathbb{N} .
$$

Then $4 k s_{k+1} \geq s_{k}$ for every $k \in \mathbb{N}$.

Proof. Clearly,

$$
4 k s_{k+1} \geq \frac{(k+1)^{2}}{k} s_{k+1} \geq \frac{(k+1)^{2}}{k} \cdot \frac{1}{e \cdot(k+1) !}=\frac{k+1}{k} \cdot \frac{1}{e \cdot k !} .
$$

On the other hand, since $k ! \cdot(k+1)^{n} \leq(k+n)$ !, we have that

$$
\begin{aligned}
\frac{k+1}{k} \cdot \frac{1}{e \cdot k !} & =\frac{1}{e \cdot k !} \cdot \frac{1}{1-1 /(k+1)} \\
& =\frac{1}{e \cdot k !}\left(1+\frac{1}{k+1}+\frac{1}{(k+1)^{2}}+\cdots\right) \geq \sum_{n=k}^{\infty} \frac{1}{e \cdot n !} .
\end{aligned}
$$

By the definition of the Kruglov operator, the function $K \chi_{[0,1]}$ has the Poisson distribution with parameter 1 . Let

$$
\psi_{0}(t):=\int_{0}^{t}\left(K \chi_{[0,1]}\right)^{*}(s) d s .
$$

It is clear that $K: L_{\infty}(0,1) \rightarrow M_{\psi_{0}}$ and $\|K\|_{L_{\infty} \rightarrow M_{\psi_{0}}}=1$. Here $M_{\psi_{0}}$ is the Marcinkiewicz space consisting of all elements $x \in S(0,1)$ such that

$$
\|x\|_{M_{\psi_{0}}}:=\sup _{0<t \leq 1} \frac{\int_{0}^{t} x^{*}(s) d s}{\psi_{0}(t)}<\infty .
$$

Lemma 11. The following inequality holds:

$$
\inf _{0<t<1-1 / e} \frac{t \psi_{0}^{\prime}(t)}{\psi_{0}(t)} \geq \frac{1}{4}
$$


Proof. Let $s_{k}$ be as in Lemma 10. Since $\psi_{0}^{\prime}=\left(K \chi_{[0,1]}\right)^{*}$ is a Poisson random variable with parameter 1 , it follows that

$$
\psi_{0}^{\prime}(t)=k \quad \text { for all } t \in\left(s_{k+1}, s_{k}\right), k \in \mathbb{N} .
$$

Therefore,

$$
\psi_{0}\left(s_{k+1}\right)=\int_{0}^{s_{k+1}} \psi_{0}^{\prime}(t) d t=\sum_{n=k+1}^{\infty} \frac{n}{e \cdot n !}=\sum_{n=k}^{\infty} \frac{1}{e \cdot n !}=s_{k}, \quad k \in \mathbb{N} .
$$

Now, let $0<t<1-1 / e$. Then $t \in\left[s_{k+1}, s_{k}\right)$ for some $k \geq 1$, and so $\psi_{0}^{\prime}(t)=k$. Since $\psi_{0}$ is concave, the function $t / \psi_{0}(t)$ increases. Therefore, by Lemma 10,

$$
\frac{t \psi_{0}^{\prime}(t)}{\psi_{0}(t)}=\frac{k t}{\psi_{0}(t)} \geq \frac{k s_{k+1}}{\psi_{0}\left(s_{k+1}\right)}=\frac{k s_{k+1}}{s_{k}} \geq \frac{1}{4} .
$$

Lemma 12. If $Y$ is a quasi-Banach symmetric space on $(0,1)$ such that the operator $K$ maps $L_{\infty}(0,1)$ into $Y$, then $Y \supset M_{\psi_{0}}$ and

$$
\|x\|_{Y} \leq 8 C(Y)\|x\|_{M_{\psi_{0}}} \cdot\|K\|_{L_{\infty} \rightarrow Y}, \quad x \in M_{\psi_{0}} .
$$

Proof. It follows from Lemma 11 that

$$
\begin{aligned}
\|x\|_{M_{\psi_{0}}} & =\sup _{0<t \leq 1}\left(\frac{1}{\psi_{0}(t)} \int_{0}^{t} x^{*}(s) d s\right) \geq \sup _{0<t<1 / 2}\left(\frac{t x^{*}(t)}{\psi_{0}(t)}\right) \\
& \geq \inf _{0<t<1 / 2}\left(\frac{t \psi_{0}^{\prime}(t)}{\psi_{0}(t)}\right) \cdot \sup _{0<t<1 / 2}\left(\frac{x^{*}(t)}{\psi_{0}^{\prime}(t)}\right) \geq \frac{1}{4} \sup _{0<t<1 / 2}\left(\frac{x^{*}(t)}{\psi_{0}^{\prime}(t)}\right) .
\end{aligned}
$$

Therefore,

$$
x^{*}(t) \leq 4\|x\|_{M_{\psi_{0}}} \psi_{0}^{\prime}(t), \quad 0<t \leq \frac{1}{2},
$$

whence

$$
x^{*}(t) \leq \sigma_{2} x^{*}(t) \leq 4\|x\|_{M_{\psi_{0}}} \sigma_{2} \psi_{0}^{\prime}(t), \quad 0<t \leq 1 .
$$

Combining the last inequality with the obvious equalities

$$
\|K\|_{L_{\infty} \rightarrow Y}=\left\|K \chi_{[0,1]}\right\|_{Y}=\left\|\psi_{0}^{\prime}\right\|_{Y}
$$

we obtain

$$
\|x\|_{Y} \leq\left\|\sigma_{2} x^{*}\right\|_{Y} \leq 4\|x\|_{M_{\psi_{0}}}\left\|\sigma_{2} \psi_{0}^{\prime}\right\|_{Y} \leq 8 C(Y)\|x\|_{M_{\psi_{0}}}\|K\|_{L_{\infty} \rightarrow Y} .
$$

In the following lemma, we use the classical notion of majorization. Let $0 \leq$ $x, y \in L_{1}(0,1)$. We write $y \prec x$ if $\int_{0}^{t} y^{*}(s) d s \leq \int_{0}^{t} x^{*}(s) d s$ for all $t \in(0,1)$ and $\int_{0}^{1} y^{*}(s) d s=\int_{0}^{1} x^{*}(s) d s$. 
Lemma 13. Let $\left\{x_{k}\right\}_{k=1}^{n}$ and $\left\{y_{k}\right\}_{k=1}^{n}, n \in \mathbb{N}$, be sequences of positive and independent functions from $L_{1}(0,1)$. If $y_{k} \prec x_{k}$ for each $k$, then

$$
\sum_{k=1}^{n} y_{k} \prec \sum_{k=1}^{n} x_{k}
$$

Proof. Define the functions $x, y \in L_{1}(0,1)$ by setting

$$
x(\omega):=\sum_{k=1}^{n} x_{k}\left(\omega_{k}\right), \quad y(\omega):=\sum_{k=1}^{n} y_{k}\left(\omega_{k}\right) .
$$

It follows from the assumption that for every $1 \leq k \leq n$ there exists a bistochastic operator $A_{k}$ (on $L_{1}(0,1)$ ) such that $A_{k} x_{k}=y_{k}$ [Bennett and Sharpley 1988, Proposition 3.2.9]. A moment's reflection shows that the operator $A:=\bigotimes_{k=1}^{n} A_{k}$ is a bistochastic operator on $L_{1}(\Omega, \mathbb{P})$ (which we identify with $L_{1}(0,1)$ ) and that $A x=\sum_{k=1}^{n} A_{k} x_{k}\left(\omega_{k}\right)$. Applying Proposition 3.2.4 of the same reference, we arrive at

$$
y=\sum_{k=1}^{n} A_{k} x_{k}\left(\omega_{k}\right)=A x \prec x .
$$

Since $\sum_{k=1}^{n} x_{k}$ (respectively, $\sum_{k=1}^{n} y_{k}$ ) is equimeasurable with $x$ (respectively, $y$ ), the assertion follows.

Proposition 14. If $\left\{x_{k}\right\}_{k=1}^{n}, n \in \mathbb{N}$, is a sequence of bounded independent functions, then

$$
\left\|\sum_{k=1}^{n} x_{k}\right\|_{M_{\psi_{0}}} \leq 2\left\|\bigoplus_{k=1}^{n} x_{k}\right\|_{L_{1} \cap L_{\infty}(0, \infty)} .
$$

Proof. Without loss of generality, we can assume that $x_{k} \geq 0$ for $1 \leq k \leq n$. Suppose that

$$
\left\|\bigoplus_{k=1}^{n} x_{k}\right\|_{\infty}=1 \quad \text { and } \quad\left\|x_{k}\right\|_{1}=\alpha_{k}
$$

If $\alpha=\sum_{k=1}^{n} \alpha_{k}>1$, then $x_{k} \prec \alpha \chi_{\left[0, \alpha^{-1} \alpha_{k}\right]}$ for $1 \leq k \leq n$. Applying Lemma 13, we obtain

$$
\sum_{k=1}^{n} x_{k} \prec \alpha \sum_{k=1}^{n} \chi_{\left[0, \alpha^{-1} \alpha_{k}\right]}\left(\omega_{k}\right) .
$$

From the definition of the norm of a Marcinkiewicz space, Proposition 9 and the equalities $\|K\|_{L_{\infty} \rightarrow M_{\psi_{0}}}=1$ and $C\left(M_{\psi_{0}}\right)=1$, we obtain 


$$
\begin{aligned}
\left\|\sum_{k=1}^{n} x_{k}\right\|_{M_{\psi_{0}}} & \leq \alpha\left\|\sum_{k=1}^{n} \chi_{\left[0, \alpha^{-1} \alpha_{k}\right]}\left(\omega_{k}\right)\right\|_{M_{\psi_{0}}} \\
& \leq 2 \alpha\left\|\bigoplus_{k=1}^{n} \chi_{\left[0, \alpha^{-1} \alpha_{k}\right]}\right\|_{\infty}=2\left\|\bigoplus_{k=1}^{n} x_{k}\right\|_{L_{1}(0, \infty)} .
\end{aligned}
$$

If $\alpha=\sum_{k=1}^{n} \alpha_{k}<1$, then $x_{k} \prec \chi_{\left[0, \alpha_{k}\right]}$ for $1 \leq k \leq n$. It follows from Lemma 13 that

$$
\sum_{k=1}^{n} x_{k} \prec \sum_{k=1}^{n} \chi_{\left[0, \alpha_{k}\right]}\left(\omega_{k}\right) \text {. }
$$

Therefore, by Proposition 9, we have

$$
\left\|\sum_{k=1}^{n} x_{k}\right\|_{M_{\psi_{0}}} \leq\left\|\sum_{k=1}^{n} \chi_{\left[0, \alpha_{k}\right]}\left(\omega_{k}\right)\right\|_{M_{\psi_{0}}} \leq 2\left\|\bigoplus_{k=1}^{n} \chi_{\left[0, \alpha_{k}\right]}\right\|_{\infty}=2 .
$$

Combining this estimate with inequality (6), we are done.

The following statement is an immediate consequence of Proposition 14 and Lemma 12.

Corollary 15. Let $Y$ be a quasi-Banach symmetric space on $(0,1)$ such that the operator $K$ maps $L_{\infty}(0,1)$ into $Y$. If $\left\{x_{k}\right\}_{k=1}^{n}, n \in \mathbb{N}$, is a sequence of bounded and independent functions, then

$$
\left\|\sum_{k=1}^{n} x_{k}\right\|_{Y} \leq 16 C(Y)\|K\|_{L_{\infty} \rightarrow Y}\left\|\bigoplus_{k=1}^{n} x_{k}\right\|_{L_{1} \cap L_{\infty}(0, \infty)} .
$$

Now, we are ready to prove the main result of this section related to the comparison of sums of independent functions and their disjoint copies in quasi-Banach symmetric function spaces.

Theorem 16. Let $X$ and $Y$ be quasi-Banach symmetric spaces on $(0,1)$ such that the operator $K$ acts boundedly from $X$ into $Y$. If $\left\{x_{k}\right\}_{k=1}^{n} \subset X, n \in \mathbb{N}$ is a sequence of independent functions, then

$$
\left\|\sum_{k=1}^{n} x_{k}\right\|_{Y} \leq 16 C^{2}(Y)\|K\|_{X \rightarrow Y}\left\|\bigoplus_{k=1}^{n} x_{k}\right\|_{Z_{X}^{1}} .
$$

Proof. Let us write $x$ for $\bigoplus_{k=1}^{n} x_{k}$. Define the functions

$$
x_{k, 1}:=x_{k} \chi_{\left\{\left|x_{k}\right|>x^{*}(1)\right\}}, \quad x_{k, 2}:=x_{k}-x_{k, 1}, \quad 1 \leq k \leq n .
$$

The functions $x_{k, 1}, 1 \leq k \leq n$, are independent, as are the functions $x_{k, 2}, 1 \leq k \leq n$. 
Moreover, it is easy to see that

$$
\bigoplus_{k=1}^{n}\left|x_{k, 1}\right| \sim x^{*} \chi_{(0,1)} \text { and } \bigoplus_{k=1}^{n}\left|x_{k, 2}\right| \sim x^{*} \chi_{(1, \infty)} .
$$

Since $L_{\infty}(0,1) \subset X$ and $\|x\|_{X} \leq\|x\|_{\infty}, x \in L_{\infty}(0,1)$, it follows from the assumption of the theorem that $K: L_{\infty}(0,1) \rightarrow Y$ and $\|K\|_{L_{\infty} \rightarrow Y} \leq\|K\|_{X \rightarrow Y}$. Therefore, applying Proposition 9 and Corollary 15, we obtain

$$
\begin{aligned}
\left\|\sum_{k=1}^{n} x_{k}\right\|_{Y} & \leq C(Y)\left(\left\|\sum_{k=1}^{n} x_{k, 1}\right\|_{Y}+\left\|\sum_{k=1}^{n} x_{k, 2}\right\|_{Y}\right) \\
& \leq 16 C^{2}(Y)\|K\|_{X \rightarrow Y}\left(\left\|\bigoplus_{k=1}^{n} x_{k, 1}\right\|_{X}+\left\|\bigoplus_{k=1}^{n} x_{k, 2}\right\|_{L_{1} \cap L_{\infty}(0, \infty)}\right) \\
& \leq 16 C^{2}(Y)\|K\|_{X \rightarrow Y}\left(\left\|x^{*} \chi_{(0,1)}\right\|_{X}+\left\|\min \left\{x^{*}, x^{*}(1)\right\}\right\|_{L_{1} \cap L_{\infty}(0, \infty)}\right) .
\end{aligned}
$$

\section{Disjointification inequalities for symmetrically distributed (mean zero) functions}

If we assume that the independent functions $x_{k}, 1 \leq k \leq n$, in the statement of Theorem 16 are symmetrically distributed, then the disjointification inequality (7) can be significantly improved. In particular, we are able to extend estimates from [Astashkin and Sukochev 2007] for symmetric Banach function spaces to the quasi-Banach setting. Our main tool is the following remarkable inequality due to Prokhorov [1959], which we restate here using the direct sum notation.

Theorem 17. If $\left\{x_{k}\right\}_{k=1}^{n}(n \in \mathbb{N})$ is a sequence of bounded independent symmetrically distributed random variables on $(0,1)$, then for all $t>0$

$$
m\left(\left\{\sum_{k=1}^{n} x_{k}>t\right\}\right) \leq \exp \left(-\frac{t}{2\left\|\bigoplus_{k=1}^{n} x_{k}\right\|_{\infty}} \operatorname{arcsinh} \frac{t\left\|\bigoplus_{k=1}^{n} x_{k}\right\|_{\infty}}{2\left\|\bigoplus_{k=1}^{n} x_{k}\right\|_{2}^{2}}\right) .
$$

Let the function $\psi_{0}$ be as in the previous section.

Proposition 18. If $\left\{x_{k}\right\}_{k=1}^{n}, n \in \mathbb{N}$, is a sequence of bounded independent symmetrically distributed functions on $(0,1)$, then

$$
\left\|\sum_{k=1}^{n} x_{k}\right\|_{M_{\psi_{0}}} \leq C_{\mathrm{abs}}\left\|\bigoplus_{k=1}^{n} x_{k}\right\|_{L_{2} \cap L_{\infty}(0, \infty)},
$$

for some absolute constant $C_{\mathrm{abs}}$. 
Proof. For every $m \geq 1$, we define a linear operator $A_{m}: L_{2} \cap L_{\infty}(0, \infty) \rightarrow M_{\psi_{0}}$ by setting for $x \in L_{2} \cap L_{\infty}(0, \infty)$

$$
A_{m} x(\omega):=\sum_{k=1}^{m} x\left(k-1+\omega_{2 k-1}\right) r\left(\omega_{2 k}\right)
$$

where $r(t)=1$ if $0 \leq t \leq \frac{1}{2}$ and $r(t)=-1$ if $\frac{1}{2}<t \leq 1$. It is clear that

$$
\left\|A_{m}\right\|_{L_{2} \cap L_{\infty} \rightarrow M_{\psi_{0}}} \leq m, \quad m \in \mathbb{N} .
$$

Our objective is to show that for every fixed $x \in L_{2} \cap L_{\infty}(0, \infty)$ the orbit $\left\{A_{m} x\right\}_{m=1}^{\infty}$ is uniformly bounded in $M_{\psi_{0}}$. Provided we have done so, the uniform boundedness principle guarantees that the sequence $\left\{\left\|A_{m}\right\|_{L_{2} \cap L_{\infty} \rightarrow M_{\psi}}\right\}_{m=1}^{\infty}$ is uniformly bounded, and the assertion of the theorem would follow from this fact since the sum $\sum_{k=1}^{n} x_{k}$ for a given sequence $\left\{x_{k}\right\}_{k=1}^{n}$ of bounded independent symmetrically distributed functions on $(0,1)$ is equidistributed with the function $A_{n} z$, where

$$
z:=\bigoplus_{k=1}^{n} x_{k}
$$

Fix $x \in L_{2} \cap L_{\infty}(0, \infty)$, and set

$$
\alpha(x):=\|x\|_{\infty}+\sup _{n} \frac{\left\|x \chi_{[0, n]}\right\|_{2}^{2}}{\left\|x \chi_{[0, n]}\right\|_{\infty}}
$$

(here, $0 / 0$ is set to be 0 ). Clearly, $\alpha(x)<\infty$ and our objective would be achieved if we show that

$$
\left\|A_{m} x\right\|_{M_{\psi_{0}}} \leq 4 e \cdot \alpha(x) \text { for all } m \in \mathbb{N} .
$$

Fix $m \in \mathbb{N}$. Since

$$
\left(\bigoplus_{k=1}^{m} x\left(k-1+\omega_{2 k-1}\right) r\left(\omega_{2 k}\right)\right)^{*}=\left(x \chi_{[0, m]}\right)^{*},
$$

it follows from (8) that for every $t>0$, we have

$$
m\left(\left\{\left|A_{m} x\right|>t \alpha(x)\right\}\right) \leq \exp \left(-\frac{t \alpha(x)}{2\left\|x \chi_{[0, m]}\right\|_{\infty}} \operatorname{arcsinh} \frac{t \alpha(x)\left\|x \chi_{[0, m]}\right\|_{\infty}}{2\left\|x \chi_{[0, m]}\right\|_{2}^{2}}\right) .
$$

Combining this estimate with the obvious inequalities

$$
\frac{t \alpha(x)}{2\left\|x \chi_{[0, m]}\right\|_{\infty}} \geq \frac{t}{2}, \quad \operatorname{arcsinh} \frac{t \alpha(x)\left\|x \chi_{[0, m]}\right\|_{\infty}}{2\left\|x \chi_{[0, m]}\right\|_{2}^{2}} \geq \operatorname{arcsinh} \frac{t}{2},
$$

we arrive at

$$
m\left(\left\{\left|A_{m} x\right|>t \alpha(x)\right\}\right) \leq \exp \left(-\frac{t}{2} \operatorname{arcsinh} \frac{t}{2}\right) .
$$


The right-hand side of the preceding inequality is in fact directly related to the distribution function of the function $\psi_{0}^{\prime}$. Indeed, in the proof of Lemma 11 we have already pointed out that $\psi_{0}^{\prime}:=\left(K \chi_{[0,1]}\right)^{*}$ is a Poisson random variable with parameter 1. A direct calculation yields the estimate

$$
m\left(\left\{\psi_{0}^{\prime}>t\right\}\right) \geq \exp (-1-2 t \cdot \operatorname{arcsinh}(2 t)), \quad t>0,
$$

which, in turn, implies

$$
m\left(\left\{4 \psi_{0}^{\prime}>t\right\}\right) \geq \exp \left(-1-\frac{t}{2} \operatorname{arcsinh} \frac{t}{2}\right), \quad t>0 .
$$

Combining this with (10), we infer

$$
m\left(\left\{\left|A_{m} x\right|>t \alpha(x)\right\}\right) \leq e \cdot m\left(\left\{4 \psi_{0}^{\prime}>t\right\}\right) .
$$

Since $\left\|\psi_{0}^{\prime}\right\|_{M\left(\psi_{0}\right)}=1$, from the preceding estimate and [Braverman 1994, Proposition 1.2], inequality (9) follows.

The corollary below follows from Proposition 18 and Lemma 12.

Corollary 19. Let $Y$ be a quasi-Banach symmetric space on $(0,1)$ such that the operator $K$ maps $L_{\infty}(0,1)$ into $Y$. If $\left\{x_{k}\right\}_{k=1}^{n}, n \in \mathbb{N}$, is a sequence of bounded independent symmetrically distributed functions, then

$$
\left\|\sum_{k=1}^{n} x_{k}\right\|_{Y} \leq 8 C_{\mathrm{abs}} C(Y)\|K\|_{L_{\infty} \rightarrow Y}\left\|\bigoplus_{k=1}^{n} x_{k}\right\|_{L_{2} \cap L_{\infty}(0, \infty)} .
$$

We need the following assertion proved by Braverman [1994, Proposition 1.11] in the Banach setting. The proof in the quasi-Banach setting is identical.

Lemma 20. If a quasi-Banach symmetric space $X$ on $(0,1)$ embeds into $L_{1}(0,1)$, then there exists a constant $C_{0}(X)$ such that

$$
\|x\|_{X} \leq C_{0}(X)\left\|x\left(\omega_{1}\right)-x\left(\omega_{2}\right)\right\|_{X}
$$

for every mean zero function $x \in X$.

We are now ready to present the main result of this section.

Theorem 21. Let $X$ and $Y$ be quasi-Banach symmetric spaces on $(0,1)$ such that $K: X \rightarrow Y$.

(a) If $\left\{x_{k}\right\}_{k=1}^{n} \subset X, n \in \mathbb{N}$, is a sequence of independent symmetrically distributed functions, then

$$
\left\|\sum_{k=1}^{n} x_{k}\right\|_{Y} \leq 8 C_{\text {abs }} C^{2}(Y)\|K\|_{X \rightarrow Y}\left\|\bigoplus_{k=1}^{n} x_{k}\right\|_{Z_{X}^{2}} .
$$


(b) If $X \subset L_{1}(0,1)$, then the inequality

$$
\left\|\sum_{k=1}^{n} x_{k}\right\|_{Y} \leq 16 C_{\mathrm{abs}} C_{0}(Y) C^{2}(Y) C(X)\|K\|_{X \rightarrow Y}\left\|\bigoplus_{k=1}^{n} x_{k}\right\|_{Z_{X}^{2}}
$$

holds for every sequence $\left\{x_{k}\right\}_{k=1}^{n}, n \in \mathbb{N}$, of independent mean zero functions from $X$.

Proof. The proof of the first assertion is similar to the proof of Theorem 16, with the only difference being that the reference to Corollary 15 should be replaced with a reference to Corollary 19.

In the proof of the second assertion we use the standard symmetrization trick. Define the functions $y_{k} \in X, 1 \leq k \leq n$, by setting

$$
y_{k}(\omega):=x_{k}\left(\omega_{2 k-1}\right)-x_{k}\left(\omega_{2 k}\right) .
$$

By Lemma 20,

$$
\left\|\sum_{k=1}^{n} x_{k}\right\|_{Y} \leq C_{0}(Y)\left\|\sum_{k=1}^{n} x_{k}\left(\omega_{2 k-1}\right)-\sum_{k=1}^{n} x_{k}\left(\omega_{2 k}\right)\right\|_{Y}=C_{0}(Y)\left\|\sum_{k=1}^{n} y_{k}\right\|_{Y} .
$$

Evidently, $y_{k}, 1 \leq k \leq n$, are independent and symmetrically distributed. Therefore, by (a), we obtain

$$
\left\|\sum_{k=1}^{n} y_{k}\right\|_{Y} \leq 8 C_{\text {abs }} C^{2}(Y)\|K\|_{X \rightarrow Y}\left\|\bigoplus_{k=1}^{n} y_{k}\right\|_{Z_{X}^{2}} .
$$

Observing that for every $t>0$, we have

$$
m\left(\left\{\left|\bigoplus_{k=1}^{n} y_{k}\right|>t\right\}\right) \leq 2 m\left(\left\{s>0:\left|\bigoplus_{k=1}^{n} x_{k}\right|>t\right\}\right)
$$

and appealing to the fact that $Z_{X}^{2}$ is a quasi-Banach symmetric space with modulus of concavity $C(X)$, we infer

$$
\left\|\bigoplus_{k=1}^{n} y_{k}\right\|_{Z_{X}^{2}} \leq 2 C(X)\left\|\bigoplus_{k=1}^{n} x_{k}\right\|_{Z_{X}^{2}} .
$$

Combining these inequalities, we conclude the proof.

\section{Khintchine inequality in quasi-Banach spaces}

In this section, we provide an extension of the classical Khintchine inequality to general quasi-Banach symmetric function spaces. We begin with the formulation of the main results of this section. 
Theorem 22. Let $X$ and $Y$ be quasi-Banach symmetric spaces on the interval $(0,1)$ such that the operator $K$ is bounded from $X$ into $Y$. If $\left\{x_{k}\right\}_{k=1}^{n}, n \in \mathbb{N}$, is a sequence of independent symmetrically distributed random variables from $X$, then

$$
\left\|\sum_{k=1}^{n} x_{k}\right\|_{Y} \leq 512 C_{\mathrm{abs}} C^{6}(X) C^{2}(Y)\|K\|_{X \rightarrow Y}\left\|\left(\sum_{k=1}^{n} x_{k}^{2}\right)^{1 / 2}\right\|_{X} .
$$

The next theorem shows that in the case when $X=Y$ the boundedness of the Kruglov operator is a necessary and sufficient condition for the inequalities of the type (13). In the Banach setting, an analogous result was earlier proved in [Astashkin 2008].

Theorem 23. Let $X$ be a quasi-Banach symmetric function space on $(0,1)$ which is separable or has the Fatou property. The following conditions are equivalent:

(a) There is a constant $C>0$ such that the inequality

$$
\left\|\sum_{k=1}^{n} x_{k}\right\|_{X} \leq C\left\|\left(\sum_{k=1}^{n} x_{k}^{2}\right)^{1 / 2}\right\|_{X}
$$

holds for every sequence $\left\{x_{k}\right\}_{k=1}^{n} \subset X, n \in \mathbb{N}$, of independent symmetrically distributed functions.

(b) $K: X \rightarrow X$.

For the proof we will need a series of lemmas. The first two of them are well known; however, we present their short proofs for the reader's convenience.

Lemma 24. Let $X$ be a quasi-Banach symmetric space on $(0,1)$. If we set $p:=$ $\frac{1}{2} \log _{2}^{-1}(2 C(X))$, then $X \subset L_{p}(0,1)$ and

$$
\|x\|_{p} \leq 8 C^{3}(X)\|x\|_{X}, \quad x \in X .
$$

Proof. Define an increasing function $\psi$ on $(0,1)$ by the formula $\psi(u):=\left\|\chi_{[0, u]}\right\|_{X}$, $0<u<1$. It follows from the definition of a quasinorm that

$$
\psi(2 u) \leq 2 C(X) \psi(u), \quad 0<u \leq 1,
$$

whence

$$
\psi\left(2^{-n}\right) \geq(2 C(X))^{-n}, \quad n \geq 0 .
$$

If $u \in(0,1]$ is arbitrary, then $u \in\left[2^{-n-1}, 2^{-n}\right]$ for some $n \geq 0$. Hence,

$$
\psi(u) \geq \psi\left(2^{-n-1}\right) \geq 2^{-(n+1) \log _{2}(2 C(X))} \geq \frac{1}{2 C(X)} u^{\log _{2}(2 C(X))} .
$$

If $x \in X$, then for every $0<t \leq 1$ we have

$$
\|x\|_{X} \geq\left\|x^{*}(t) \chi_{[0, t]}\right\|_{X} \geq x^{*}(t) \frac{1}{2 C(X)} t^{\log _{2}(2 C(X))} .
$$


Hence,

$$
x^{*}(t) \leq 2\|x\|_{X} C(X) t^{-\log _{2}(2 C(X))}, \quad 0<t \leq 1 .
$$

The assertion follows immediately.

Lemma 25. If $0<p<1$ and $x, y \in L_{1}(0,1)$ are positive, then from $y \prec x$ it follows that $\|y\|_{p} \geq\|x\|_{p}$.

Proof. Fix $\varepsilon>0$. Passing to step-function approximation, we easily infer that there exist $n \in \mathbb{N}$ and a function

$$
z:=\sum_{k=1}^{n} \lambda_{k} x_{k} \quad \text { with } \quad x_{k} \geq 0, x_{k}^{*}=x^{*} \text { and } \sum_{k=1}^{n} \lambda_{k}=1, \lambda_{k} \geq 0,
$$

such that $\|y-z\|_{1} \leq \varepsilon$. It follows now from the Minkowski inequality that

$$
\|z\|_{p}=\left\|\sum_{k=1}^{n} \lambda_{k} x_{k}\right\|_{p} \geq \sum_{k=1}^{n} \lambda_{k}\left\|x_{k}\right\|_{p}=\|x\|_{p} .
$$

Since $\varepsilon>0$ is arbitrarily small and the quasinorm in $L_{p}(0,1), 0<p<1$, is continuous with respect to $L_{1}$-convergence, the proof is complete.

Lemma 26. Let $0<p<1$ and let $\left\{y_{k}\right\}_{k=1}^{n}, n \in \mathbb{N}$, be a sequence of positive bounded independent functions on $(0,1)$. We have

$$
\left\|\bigoplus_{k=1}^{n} y_{k}\right\|_{1} \leq 2^{1 / p} \max \left\{\sup _{1 \leq k \leq n}\left\|y_{k}\right\|_{\infty},\left\|\sum_{k=1}^{n} y_{k}\right\|_{p}\right\} .
$$

Proof. Without loss of generality, we can assume that

$$
\sup _{1 \leq k \leq n}\left\|y_{k}\right\|_{\infty}=1, \quad\left\|y_{k}\right\|_{1}=\alpha_{k}, \quad 1 \leq k \leq n .
$$

Let $\alpha=\sum_{k=1}^{n} \alpha_{k}$. If $\alpha \leq 1$, then the assertion is evident. If $\alpha \geq 1$, then

$$
y_{k} \prec \alpha \chi_{\left[0, \alpha^{-1} \alpha_{k}\right]}, \quad 1 \leq k \leq n .
$$

From Lemma 13 it follows that

$$
\sum_{k=1}^{n} y_{k} \prec \alpha \sum_{k=1}^{n} \chi_{\left[0, \alpha^{-1} \alpha_{k}\right]}\left(\omega_{k}\right)
$$

whence, according to Lemma 25, we have

$$
\left\|\sum_{k=1}^{n} y_{k}\right\|_{p} \geq \alpha\left\|\sum_{k=1}^{n} \chi_{\left[0, \alpha^{-1} \alpha_{k}\right]}\left(\omega_{k}\right)\right\|_{p} .
$$


Combining this inequality with [Johnson and Schechtman 1989, Lemma 3], we infer

$$
2 C(p)\left\|\sum_{k=1}^{n} y_{k}\right\|_{p} \geq \alpha\left\|\bigoplus_{k=1}^{n} \chi_{\left[0, \alpha^{-1} \alpha_{k}\right]}\right\|_{p}=\alpha .
$$

Since $C(p)=2^{1 / p-1}$ for $0<p<1$, the assertion follows.

Lemma 27. Let $X$ be a quasi-Banach symmetric space on $(0,1)$. If $\left\{x_{k}\right\}_{k=1}^{n} \subset X$, $n \in \mathbb{N}$, is a sequence of bounded independent functions, then

$$
\left\|\bigoplus_{k=1}^{n} x_{k}\right\|_{2} \leq 32 C^{5}(X) \max \left\{\sup _{1 \leq k \leq n}\left\|x_{k}\right\|_{\infty},\left\|\left(\sum_{k=1}^{n} x_{k}^{2}\right)^{1 / 2}\right\|_{X}\right\} .
$$

Proof. If $p=\frac{1}{2} \log _{2}^{-1}(2 C(X))$, then by Lemma 24 we have

$$
8 C^{3}(X)\left\|\left(\sum_{k=1}^{n} x_{k}^{2}\right)^{1 / 2}\right\|_{X} \geq\left\|\left(\sum_{k=1}^{n} x_{k}^{2}\right)^{1 / 2}\right\|_{p}=\left\|\sum_{k=1}^{n} x_{k}^{2}\right\|_{p / 2}^{1 / 2} .
$$

Clearly,

$$
\left\|\bigoplus_{k=1}^{n} x_{k}\right\|_{2}=\left\|\bigoplus_{k=1}^{n} x_{k}^{2}\right\|_{1}^{1 / 2} \text { and }\left\|x_{k}\right\|_{\infty}=\left\|x_{k}^{2}\right\|_{\infty}^{1 / 2}
$$

Now, applying Lemma 26 to the functions $y_{k}=x_{k}^{2}, 1 \leq k \leq n$, we obtain the result.

Lemma 28. Let $X$ be a quasi-Banach symmetric space on $(0,1)$. If $\left\{x_{k}\right\}_{k=1}^{n} \subset X$, $n \in \mathbb{N}$, is a sequence of independent functions and if $x:=\bigoplus_{k=1}^{n} x_{k}$, then

$$
2 C(X)\left\|\left(\sum_{k=1}^{n} x_{k}^{2}\right)^{1 / 2}\right\|_{X} \geq x^{*}(1) .
$$

Proof. A simple argument shows that it is sufficient to consider the case when

$$
\sum_{k=1}^{n} m\left(\operatorname{supp}\left(x_{k}\right)\right)=1
$$

Since $\left|x_{k}\right| \geq x^{*}(1) \chi_{\operatorname{supp}\left(x_{k}\right)}, 1 \leq k \leq n$, we have

$$
\sum_{k=1}^{n} x_{k}^{2} \geq\left(x^{*}(1)\right)^{2} \sum_{k=1}^{n} \chi_{\operatorname{supp}\left(x_{k}\right)} .
$$


Since the functions $x_{k}, 1 \leq k \leq n$, are independent, the support of the function at the right-hand side of the inequality above has Lebesgue measure equal to

$$
1-\prod_{k=1}^{n}\left(1-m\left(\operatorname{supp}\left(x_{k}\right)\right)\right)
$$

which is bigger than $\frac{1}{2}$ (thanks to the condition (15) and to the arithmetic-geometric mean inequality). Therefore, since $\left\|\chi_{(0,1)}\right\|_{X}=1$, we obtain

$$
\left\|\left(\sum_{k=1}^{n} x_{k}^{2}\right)^{1 / 2}\right\|_{X} \geq x^{*}(1)\left\|\chi_{[0,1 / 2]}\right\|_{X} \geq \frac{x^{*}(1)}{2 C(X)},
$$

and the proof is complete.

Let $1 \leq p<\infty$ and let $X$ be a quasi-Banach symmetric function space on $(0,1)$ or $(0, \infty)$. The $p$-concavification of $X, X^{1 / p}$, is defined by

$$
X^{1 / p}:=\left\{x \in S(0,1)(\text { or } S(0, \infty)):|x|^{1 / p} \in X\right\}, \quad\|x\|_{X^{1 / p}}:=\left\||x|^{1 / p}\right\|_{X}^{p} .
$$

Note that the space $X^{1 / p}$, equipped with the quasinorm $\|\cdot\|_{X^{1 / p}}$, is also a quasiBanach symmetric function space (see, for instance, [Lindenstrauss and Tzafriri 1979]).

We are now ready to prove the main result of this section.

Proof of Theorem 22. Setting $x:=\bigoplus_{k=1}^{n} x_{k}$, by Theorem 21, we have

$$
\left\|\sum_{k=1}^{n} x_{k}\right\|_{Y} \leq 8 C_{\text {abs }} C^{2}(Y)\|K\|_{X \rightarrow Y}\left(\left\|x^{*} \chi_{(0,1)}\right\|_{X}+\left\|x^{*} \chi_{(1, \infty)}\right\|_{2}\right) .
$$

Arguing in the same way as in the proof of Theorem 16, we can define two sequences of independent functions $\left\{x_{k, 1}\right\}$ and $\left\{x_{k, 2}\right\}$ such that $x_{1 k}+x_{2 k}=x_{k}$, $\left|x_{k, 1}\right| \leq\left|x_{k}\right|,\left|x_{k, 2}\right| \leq\left|x_{k}\right|$, for $1 \leq k \leq n$, and the disjoint sums $\bigoplus_{k=1}^{n}\left|x_{k, 1}\right|$ and $\bigoplus_{k=1}^{n}\left|x_{k, 2}\right|$ are equimeasurable with the functions $x^{*} \chi_{(0,1)}$ and $x^{*} \chi_{(1, \infty)}$, respectively. Applying Lemma 27 to the sequence $\left\{x_{k, 2}\right\}_{k=1}^{n}$, we obtain

$$
\begin{aligned}
\left\|x^{*} \chi_{(1, \infty)}\right\|_{2} & =\left\|\bigoplus_{k=1}^{n} x_{k, 2}\right\|_{2} \\
& \leq 32 C^{5}(X) \max \left\{\sup _{1 \leq k \leq n}\left\|x_{k, 2}\right\|_{\infty},\left\|\left(\sum_{k=1}^{n} x_{k, 2}^{2}\right)^{1 / 2}\right\|_{X}\right\} .
\end{aligned}
$$

Note that $\left\|x_{k, 2}\right\|_{\infty} \leq x^{*}$ (1) for $1 \leq k \leq n$. Using Lemma 28, we obtain

$$
\left\|x^{*} \chi_{(1, \infty)}\right\|_{2} \leq 64 C^{6}(X)\left\|\left(\sum_{k=1}^{n} x_{k}^{2}\right)^{1 / 2}\right\|_{X} .
$$


On the other hand,

$$
\left\|x^{*} \chi_{(0,1)}\right\|_{X}=\left\|\bigoplus_{k=1}^{n} x_{k, 1}\right\|_{X}=\left\|\bigoplus_{k=1}^{n} x_{k, 1}^{2}\right\|_{X^{1 / 2}}^{1 / 2},
$$

and

$$
\left\|\left(\sum_{k=1}^{n} x_{k}^{2}\right)^{1 / 2}\right\|_{X} \geq\left\|\left(\sum_{k=1}^{n} x_{k, 1}^{2}\right)^{1 / 2}\right\|_{X}=\left\|\sum_{k=1}^{n} x_{k, 1}^{2}\right\|_{X^{1 / 2}}^{1 / 2} .
$$

Applying [Johnson and Schechtman 1989, Lemma 3] to the space $X^{1 / 2}$ and the functions $x_{k, 1}^{2}$, we obtain

$$
\left\|x^{*} \chi_{(0,1)}\right\|_{X} \leq\left(2 C\left(X^{1 / 2}\right)\right)^{1 / 2}\left\|\left(\sum_{k=1}^{n} x_{k}^{2}\right)^{1 / 2}\right\|_{X} .
$$

Since $C\left(X^{1 / 2}\right) \leq 4 C^{2}(X)$, the assertion follows now from the last inequality and inequalities (16) and (17).

Lemma 29. Let $x \in S(0,1), x \geq 0$, and let $n \in \mathbb{N}$. If $x_{k}, k=1,2, \ldots, 2 n$, are independent copies of the function $\sigma_{1 / n} x$, then for all sufficiently large $n \in \mathbb{N}$ we have

$$
\left(\sum_{k=1}^{n} x_{2 k}\right)^{*} \leq \sigma_{3}\left(\sum_{k=1}^{2 n}(-1)^{k} x_{k}\right)^{*}
$$

Proof. It is clear that the functions $x_{2 k-1}-x_{2 k}, 1 \leq k \leq n$, are independent. Therefore,

$$
\begin{aligned}
m\left(\left\{\sum_{k=1}^{n} x_{2 k}-x_{2 k-1}>t\right\}\right) & \geq m\left(\left\{\sum_{k=1}^{n} x_{2 k}>t, \sum_{k=1}^{n} x_{2 k-1}=0\right\}\right) \\
& =m\left(\left\{\sum_{k=1}^{n} x_{2 k}>t\right\}\right) \cdot m\left(\left\{\sum_{k=1}^{n} x_{2 k-1}=0\right\}\right) \\
& =\left(1-\frac{1}{n}\right)^{n} m\left(\left\{\sum_{k=1}^{n} x_{2 k}>t\right\}\right) .
\end{aligned}
$$

Hence, for all sufficiently large $n \in \mathbb{N}$,

$$
m\left(\left\{\left|\sum_{k=1}^{n} x_{2 k-1}-x_{2 k}\right|>t\right\}\right) \geq \frac{1}{3} m\left(\left\{\sum_{k=1}^{n} x_{2 k}>t\right\}\right) .
$$


Proof of Theorem 23. We have to prove only the implication (a) $\Longrightarrow$ (b).

Let $x \in X, x \geq 0$, and $n \in \mathbb{N}$. Taking for $x_{k}, k=1,2, \ldots, 2 n$, independent copies of the function $\sigma_{1 / n} x$, by Lemma 29 we have

$$
\left\|\sum_{k=1}^{n} x_{2 k}\right\|_{X} \leq\left\|\sigma_{3}\left(\sum_{k=1}^{2 n}(-1)^{k} x_{k}\right)\right\|_{X} \leq 3 C(X)^{2}\left\|\sum_{k=1}^{2 n}(-1)^{k} x_{k}\right\|_{X} .
$$

On the other hand, the functions $x_{2 k-1}-x_{2 k}, 1 \leq k \leq n$, are independent and symmetrically distributed. Therefore, by the assumption, we have

$$
\begin{aligned}
\left\|\sum_{k=1}^{2 n}(-1)^{k} x_{k}\right\|_{X} & \leq C\left\|\left(\sum_{k=1}^{n}\left(x_{2 k-1}-x_{2 k}\right)^{2}\right)^{1 / 2}\right\|_{X} \\
& \leq C\left\|\left(\sum_{k=1}^{n} x_{2 k-1}^{2}\right)^{1 / 2}+\left(\sum_{k=1}^{n} x_{2 k}^{2}\right)^{1 / 2}\right\|_{X} \\
& \leq 2 C \cdot C(X)\left\|\left(\sum_{k=1}^{n} x_{2 k}^{2}\right)^{1 / 2}\right\|_{X} .
\end{aligned}
$$

Combining these inequalities, we obtain

$$
\begin{aligned}
\left\|\sum_{k=1}^{n} x_{2 k}\right\| & \leq 6 C \cdot C(X)^{3}\left\|\left(\sum_{k=1}^{n} x_{2 k}^{2}\right)^{1 / 2}\right\|_{X} \\
& \leq 6 C \cdot C(X)^{3}\left\|\left(\max _{1 \leq k \leq n} x_{2 k} \cdot \sum_{k=1}^{n} x_{2 k}\right)^{1 / 2}\right\|_{X} .
\end{aligned}
$$

It follows now from Lemma 2 that

$$
\left\|\sum_{k=1}^{n} x_{2 k}\right\|_{X} \leq 6 C \cdot C(X)^{4}\left\|\max _{1 \leq k \leq n} x_{2 k}\right\|_{X}^{1 / 2} \cdot\left\|\sum_{k=1}^{n} x_{2 k}\right\|_{X}^{1 / 2} .
$$

Hence,

$$
\left\|\sum_{k=1}^{n} x_{2 k}\right\|_{X} \leq 36 C^{2} C(X)^{8}\left\|\max _{1 \leq k \leq n} x_{2 k}\right\|_{X} \leq 36 C^{2} C(X)^{8}\left\|\bigoplus_{k=1}^{n} x_{2 k}\right\|_{X} .
$$

Appealing to the definition of $x_{k}, 1 \leq k \leq 2 n$, we obtain

$$
\left(\bigoplus_{k=1}^{n} x_{2 k}\right)^{*}=x^{*} \text { and }\left(\sum_{k=1}^{n} x_{2 k}\right)^{*}=\left(H_{n} x\right)^{*},
$$

where the operator $H_{n}$ is defined by (3). 
Recall that, by Lemma $6,\left(H_{n} x\right)^{*} \rightarrow(K x)^{*}$ almost everywhere on $(0,1)$. Therefore, if $X$ has the Fatou property, it follows that $\|K x\|_{X} \leq 36 C^{2} C(X)^{8}\|x\|_{X}$, and the proof in this case is complete. If $X$ is separable, we can repeat almost verbatim the arguments used in the second part of the proof of Theorem 7.

\section{Von Bahr-Esseen type inequalities}

We have the following remarkable theorem.

Theorem 30 [von Bahr and Esseen 1965, Theorem 2]. If $1 \leq p \leq 2$ and $\left\{f_{k}\right\}_{k=1}^{n} \subset$ $L_{p}(0,1), n \in \mathbb{N}$, is a sequence of independent mean zero functions, then

$$
\left\|\sum_{k=1}^{n} f_{k}\right\|_{p} \leq\left(2 \sum_{k=1}^{n}\left\|f_{k}\right\|_{p}^{p}\right)^{1 / p} .
$$

In [Braverman 1994, § II,2], Theorem 30 is extended to Banach symmetric function spaces with the Kruglov property. Versions of disjointification inequalities obtained in Sections 3 and 4 for quasi-Banach symmetric spaces allow us to extend Braverman's result to the quasi-Banach setting. Moreover, we shall consider different quasinorms at the left- and right-hand sides of (18). Our proofs appear to be more straightforward (and simpler) than the proofs for the special case considered in [Braverman 1994].

Definition 31. Quasi-Banach symmetric function spaces $X$ and $Y$ (in this order) satisfy the von Bahr-Esseen $r$-estimate (written $\left.(X, Y) \in(B E)_{r}\right)$ if there exists a constant $B>0$ such that

$$
\left\|\sum_{k=1}^{n} f_{k}\right\|_{Y} \leq B\left(\sum_{k=1}^{n}\left\|f_{k}\right\|_{X}^{r}\right)^{1 / r}
$$

for every sequence of independent symmetrically distributed functions $\left\{f_{k}\right\}_{k=1}^{n} \subset X$, $n \in \mathbb{N}$. If, in addition, $X=Y$, then we say that $X$ satisfies the von Bahr-Esseen $r$-estimate (written $X \in(B E)_{r}$ ).

In view of this definition, we may restate Theorem 30 as $L_{p}(0,1) \in(B E)_{p}$.

Remark 32. If $Y \subset L_{1}(0,1)$, then an application of Lemma 20 yields the estimate (19) for all mean zero independent functions.

Clearly, $(X, Y) \in(B E)_{r}$ implies that $X \subset Y$. Taking Rademacher functions (see Section 1) as the $f_{k}$, it is easy to see that we always have $0<r \leq 2$. Finally, if $X$ is $p$-normed, then $p \leq r \leq 2$. 
Recall that a quasi-Banach lattice $X$ satisfies an upper $r$-estimate, $r>0$, if there is a constant $C>0$ such that

$$
\left\|\sum_{k=1}^{n} x_{k}\right\|_{X} \leq C\left(\sum_{k=1}^{n}\left\|x_{k}\right\|_{X}^{r}\right)^{1 / r}
$$

for every sequence of mutually disjoint elements $\left\{x_{k}\right\}_{k=1}^{n} \subset X, n \in \mathbb{N}$.

Recall also that a quasi-Banach symmetric space $L_{r, \infty}, r>0$, consists of all $x \in S(0,1)$ such that

$$
\|x\|_{r, \infty}:=\sup _{0<t \leq 1} x^{*}(t) t^{1 / r}<\infty .
$$

Theorem 33. Let $0<r<2$. For all quasi-Banach symmetric function spaces $X$ and $Y$ the following statements hold:

(a) If $K: X \rightarrow Y$ and $X$ satisfies an upper $r$-estimate, then $(X, Y) \in(B E)_{r}$.

(b) If $K: Y \rightarrow Y$ and, for some $C>0$ and for every sequence of mutually disjoint functions $\left\{f_{k}\right\}_{k=1}^{n} \subset X(n \in \mathbb{N})$, we have

$$
\left\|\sum_{k=1}^{n} f_{k}\right\|_{Y} \leq C\left(\sum_{k=1}^{n}\left\|f_{k}\right\|_{X}^{r}\right)^{1 / r},
$$

then $(X, Y) \in(B E)_{r}$.

(c) If $(X, Y) \in(B E)_{r}$, then (20) holds for every sequence of mutually disjoint functions $\left\{f_{k}\right\}_{k=1}^{n} \subset X, n \in \mathbb{N}$.

The main part of the proof of Theorem 33 is given below in Lemma 36.

Let $0<p<r<2$ and let $r>1$. Recall that $L_{r, \infty}$ satisfies an upper $r$-estimate (see, for example, [Braverman 1994, Theorem 1.12]) and that $K: L_{r, \infty} \rightarrow L_{r, \infty}$ by Theorem 1.3 of the same reference. Setting $X=L_{r, \infty}$ and $Y=L_{p}(0,1)$ and taking into account Remark 32, we obtain the well-known Esseen-Janson theorem (see [Esseen and Janson 1985, Theorem 4]). It is worth noting that, in contrast to the previous reference, we do not require that the functions $f_{k}$ are equidistributed.

Lemma 34. Let $r>0$ and let $X$ and $Y$ be quasi-Banach symmetric function spaces. Suppose that there is a constant $C>0$ such that for every sequence of mutually disjoint functions $\left\{f_{k}\right\}_{k=1}^{n} \subset X, n \in \mathbb{N}$, inequality (20) holds. Then $X \subset L_{r, \infty}$.

Proof. Fix $t \in(0,1]$ and let $n \in \mathbb{N}$ be such that $1 / 2<n t \leq 1$. Since $\chi_{(0, t n)}=$ $\sum_{k=1}^{n} \chi_{(t(k-1), t k)}$, the functions $\varphi_{X}(t):=\left\|\chi_{(0, t)}\right\|_{X}$ and $\varphi_{Y}(t):=\left\|\chi_{(0, t)}\right\|_{Y}$ satisfy the estimate

$$
\varphi_{Y}(t n) \leq C\left(\sum_{k=1}^{n}\|\chi(t(k-1), t k)\|_{X}^{r}\right)^{1 / r}=C \varphi_{X}(t) n^{1 / r},
$$


by (20). Hence, we obtain that

$$
\varphi_{X}(t) \geq C^{-1} \varphi_{Y}(t n) n^{-1 / r} \geq C^{-1} \varphi_{Y}(1 / 2) t^{1 / r}=C_{1}^{-1} t^{1 / r},
$$

whence for every $x \in X$

$$
\|x\|_{X} \geq x^{*}(t)\left\|\chi_{(0, t)}\right\|_{X}=x^{*}(t) \varphi_{X}(t) \geq C_{1}^{-1} x^{*}(t) t^{1 / r}, \quad 0<t \leq 1 .
$$

Therefore, $\|x\|_{r, \infty} \leq C_{1}\|x\|_{X}$ for all $x \in X$ and the proof is completed.

Lemma 35. Let $X$ be a quasi-Banach symmetric function space on $(0,1)$ satisfying an upper $r$-estimate, $0<r<2$. There exists $C_{X}>0$ such that for every sequence $\left\{x_{k}\right\}_{k=1}^{\infty} \subset X$ we have

$$
\left\|\bigoplus_{k=1}^{\infty} x_{k}\right\|_{Z_{L_{r, \infty}^{2}}^{2}} \leq C_{X}\left(\sum_{k=1}^{\infty}\left\|x_{k}\right\|_{X}^{r}\right)^{1 / r}
$$

Proof. By Lemma 34, we have $X \subset L_{r, \infty}$. Therefore, $x_{k}^{*} \leq\left\|x_{k}\right\|_{r, \infty} \xi_{r}$, where $\xi_{r}(t)=t^{-1 / r}, 0<t \leq 1$, whence

$$
\left\|\bigoplus_{k=1}^{\infty} x_{k}\right\|_{Z_{L r, \infty}^{2}} \leq C\left\|\bigoplus_{k=1}^{\infty}\right\| x_{k}\left\|_{r, \infty} \xi_{r}\right\|_{Z_{L_{r, \infty}^{2}}^{2}}
$$

Note that for any $a_{k} \geq 0$ we have

$$
\bigoplus_{k=1}^{\infty} a_{k} \xi_{r} \sim\left(\sum_{k=1}^{\infty} a_{k}^{r}\right)^{1 / r} \xi_{r}
$$

Hence,

$\left\|\bigoplus_{k=1}^{\infty} x_{k}\right\|_{Z_{L r, \infty}^{2}} \leq C\left(\sum_{k=1}^{\infty}\left\|x_{k}\right\|_{r, \infty}^{r}\right)^{1 / r}\left\|\xi_{r}\right\|_{Z_{L_{r}, \infty}^{2}} \leq C^{\prime}\left\|\xi_{r}\right\|_{Z_{L r, \infty}^{2}}\left(\sum_{k=1}^{\infty}\left\|x_{k}\right\|_{X}^{r}\right)^{1 / r}$, and the result follows.

Lemma 36. Let $X$ be a quasi-Banach symmetric function space on $(0,1)$ satisfying an upper $r$-estimate, $0<r<2$. There exists a constant $B_{X}>0$ such that for every sequence $\left\{x_{k}\right\}_{k=1}^{\infty} \subset X$ we have

$$
\left\|\bigoplus_{k=1}^{\infty} x_{k}\right\|_{Z_{X}^{2}} \leq B_{X}\left(\sum_{k=1}^{\infty}\left\|x_{k}\right\|_{X}^{r}\right)^{1 / r}
$$

Proof. By the definition of the quasinorm in $Z_{X}^{2}$, we have that

$$
\|z\|_{Z_{X}^{2}} \leq\left\|z^{*} \chi_{(0,1)}\right\|_{X}+\|z\|_{Z_{L_{r, \infty}}^{2}}, \quad z \in Z_{X}^{2} .
$$


Denote $\bigoplus_{k=1}^{\infty}\left|x_{k}\right|$ by $x$, for brevity. Without loss of generality, we can assume that $x^{*}$ does not have any interval of constancy. Setting $y_{k}=x_{k} \chi_{\left\{\left|x_{k}\right|>x^{*}(1)\right\}}$, we have

$$
\bigoplus_{k=1}^{\infty}\left|y_{k}\right| \sim x^{*} \chi_{(0,1)}
$$

Therefore, since $X$ satisfies an upper $r$-estimate, we obtain

$$
\left\|x^{*} \chi_{(0,1)}\right\|_{X}=\left\|\bigoplus_{k=1}^{\infty} y_{k}\right\|_{X} \leq C\left(\sum_{k=1}^{\infty}\left\|y_{k}\right\|_{X}^{r}\right)^{1 / r} \leq C\left(\sum_{k=1}^{\infty}\left\|x_{k}\right\|_{X}^{r}\right)^{1 / r} .
$$

The assertion follows now from inequality (21) and the preceding lemma.

Proof of Theorem 33. The first assertion follows from Theorem 21 and Lemma 36. The proof of the second assertion is identical.

Now, we prove the third assertion. Suppose that $(X, Y) \in(B E)_{r}$. Let the functions $f_{k} \in X, 1 \leq k \leq n$, be pairwise disjoint and let $g_{k}, 1 \leq k \leq n$, be their independent copies. Without loss of generality, we can assume that the $f_{k}$ (and therefore the $g_{k}$ as well) are symmetrically distributed. By [Johnson and Schechtman 1989, Theorem 1], we have

$$
\left\|\sum_{k=1}^{n} f_{k}\right\|_{Y}=\left\|\sum_{k=1}^{n} f_{k}\right\|_{Z_{Y}^{2}} \leq C^{\prime}\left\|\sum_{k=1}^{n} g_{k}\right\|_{Y} \leq C^{\prime} B\left(\sum_{k=1}^{n}\left\|f_{k}\right\|_{X}^{r}\right)^{1 / r},
$$

which is (20) with $C=C^{\prime} B$.

If $X=Y$, then estimate (20) means that $X$ satisfies an upper $r$-estimate and we obtain the following corollary.

Corollary 37. Let $0<r<2$ and let $X$ be a quasi-Banach symmetric function space such that $K: X \rightarrow X$. Then $X \in(B E)_{r}$ if and only if $X$ satisfies an upper $r$-estimate.

In the Banach-space setting this result may be found in [Braverman 1994, Theorem 2.3].

For $r=2$, we have the following result.

Theorem 38. Let $X$ and $Y$ be quasi-Banach symmetric function spaces.

(a) Suppose that $X \supset L_{2}(0,1)$. If $K: X \rightarrow Y$ and $X$ satisfies an upper 2-estimate, or if $K: Y \rightarrow Y$ and for some $C>0$ and for every sequence of mutually disjoint functions $\left\{f_{k}\right\}_{k=1}^{n} \subset X, n \in \mathbb{N}$, we have

$$
\left\|\sum_{k=1}^{n} f_{k}\right\|_{Y} \leq C\left(\sum_{k=1}^{n}\left\|f_{k}\right\|_{X}^{2}\right)^{1 / 2},
$$

then $(X, Y) \in(B E)_{2}$. 
(b) If $(X, Y) \in(B E)_{2}$, then $X \supset L_{2}(0,1)$ and inequality (22) holds for some $C>0$ and for every sequence of mutually disjoint functions $\left\{f_{k}\right\}_{k=1}^{n} \subset X$, $n \in \mathbb{N}$.

Proof. (a) The proof is identical to that of the preceding theorem, substituting the reference to Lemma 36 with the reference to the following assertion.

Lemma 39. Let a quasi-Banach symmetric space $X$ satisfy an upper 2-estimate and let $X \supset L_{2}(0,1)$. There exists a constant $B_{X}>0$ such that for every sequence $\left\{x_{k}\right\}_{k=1}^{\infty} \subset X$ we have

$$
\left\|\bigoplus_{k=1}^{\infty} x_{k}\right\|_{Z_{X}^{2}} \leq B_{X}\left(\sum_{k=1}^{\infty}\left\|x_{k}\right\|_{X}^{2}\right)^{1 / 2}
$$

(b) Inequality (22) can be proved in exactly the same way as in Theorem 33. Therefore, it remains to show that $X \subset L_{2}(0,1)$.

Let $f \in X$ be symmetrically distributed and let $\left\{f_{k}\right\}_{k=1}^{\infty}$ be a sequence of its independent copies. By assumption, $(X, Y) \in(B E)_{2}$ and, therefore,

$$
\left\|n^{-1 / 2} \sum_{k=1}^{n} f_{k}\right\|_{Y} \leq C\left(n^{-1} \sum_{k=1}^{n}\left\|f_{k}\right\|_{X}^{2}\right)^{1 / 2}=C\|f\|_{X}, \quad n=1,2, \ldots
$$

By Lemma 24, there exists $p>0$ such that $Y \subset L_{p}(0,1)$. Hence, by the previous inequality, we have

$$
\sup _{n \geq 1} \int_{0}^{1}\left|n^{-1 / 2} \sum_{k=1}^{n} f_{k}(t)\right|^{p} d t<\infty .
$$

Applying [Esseen and Janson 1985, Theorem 2], we obtain that $f \in L_{2}(0,1)$. Since both $X$ and $L_{2}(0,1)$ are symmetric, the assertion follows.

Corollary 40. Let $X$ be a quasi-Banach symmetric space such that $K: X \rightarrow X$. Then $X \in(B E)_{2}$ if and only if $X$ satisfies an upper 2-estimate and $X \subset L_{2}(0,1)$.

This assertion was proved by Braverman [1994, Theorem 2.4] in the Banach setting.

Remark 41. Though the condition $K: X \rightarrow X$ is essential in both Theorem 33 and Theorem 38, it is not necessary. For example, Exp $L_{2} \in(B E)_{2}$ [Braverman 1994, Theorem 2.9], but $K: \operatorname{Exp} L_{2} \nrightarrow \nrightarrow \operatorname{Exp} L_{2}$.

\section{References}

[Astashkin 2008] S. V. Astashkin, "Независимые функции в симметричных пространствах и свойство Круглова", Mat. Sb. 199:7 (2008), 3-20. Translated as "Independent functions in symmetric spaces and the Kruglov property" in Sb. Math. 199:7 (2008), 945-963. MR 2010b: $46055 \mathrm{Zbl} 1280.46015$ 
[Astashkin and Sukochev 2005] S. V. Astashkin and F. A. Sukochev, "Series of independent random variables in rearrangement invariant spaces: an operator approach", Israel J. Math. 145 (2005), 125-156. MR 2006d:60012 Zbl 1084.46020

[Astashkin and Sukochev 2007] S. V. Astashkin and F. A. Sukochev, "Ряды независимых функций с нулевым средним в симметричных пространствах со свойством Круглова", Zap. Nauchn. Sem. (POMI) 345:35 (2007), 25-50. Translated as "Series of zero-mean independent functions in symmetric spaces with the Kruglov property" in J. Math. Sci. (NY) 148:6 (2008), 795-809. MR 2010j:60103

[Astashkin and Sukochev 2010] S. V. Astashkin and F. A. Sukochev, "Независимые функции и геометрия Банаховых пространств", Uspekhi Mat. Nauk 65:6(396) (2010), 3-86. Translated as "Independent functions and the geometry of Banach spaces" in Russian Math. Surveys 65:6 (2010), 1003-1081. MR 2012b:46002 Zbl 1219.46025

[Astashkin et al. 2011] S. V. Astashkin, F. A. Sukochev, and C. P. Wong, "Disjointification of martingale differences and conditionally independent random variables with some applications", Stud. Math. 205:2 (2011), 171-200. MR 2012h:60134 Zbl 1238.46009

[von Bahr and Esseen 1965] B. von Bahr and C.-G. Esseen, "Inequalities for the $r$ th absolute moment of a sum of random variables, $1 \leq r \leq 2$ ", Ann. Math. Statist 36 (1965), 299-303. MR 30 \#645 Zbl 0134.36902

[Bennett and Sharpley 1988] C. Bennett and R. Sharpley, Interpolation of operators, Pure and Applied Mathematics 129, Academic Press, Boston, 1988. MR 89e:46001 Zbl 0647.46057

[Borovkov 1998] A. A. Borovkov, Probability theory, Gordon and Breach, Amsterdam, 1998. MR 2000f:60001 Zbl 0918.60003

[Braverman 1994] M. S. Braverman, Independent random variables and rearrangement invariant spaces, London Mathematical Society Lecture Note Series 194, Cambridge University Press, 1994. MR 95j:60027 Zbl 0817.46031

[Esseen and Janson 1985] C.-G. Esseen and S. Janson, "On moment conditions for normed sums of independent variables and martingale differences", Stochastic Process. Appl. 19:1 (1985), 173-182. MR 86m:60120 Zbl 0554.60050

[Johnson and Schechtman 1988] W. B. Johnson and G. Schechtman, "Martingale inequalities in rearrangement invariant function spaces", Israel J. Math. 64:3 (1988), 267-275. MR 90g:60048 Zbl 0672.60049

[Johnson and Schechtman 1989] W. B. Johnson and G. Schechtman, "Sums of independent random variables in rearrangement invariant function spaces", Ann. Probab. 17:2 (1989), 789-808. MR 90h:60045 Zbl 0674.60051

[Johnson et al. 1979] W. B. Johnson, B. Maurey, G. Schechtman, and L. Tzafriri, Symmetric structures in Banach spaces, Memoirs of the American Mathematical Society 19:217, American Mathematical Society, Providence, RI, 1979. MR 82j:46025 Zbl 0421.46023

[Kalton et al. 1984] N. J. Kalton, N. T. Peck, and J. W. Roberts, An F-space sampler, London Mathematical Society Lecture Note Series 89, Cambridge University Press, 1984. MR 87c:46002 Zbl 0556.46002

[Khintchine 1923] A. Khintchine, “Über dyadische Brüche”, Math. Z. 18:1 (1923), 109-116. MR 1544623 JFM 49.0132.01

[Kruglov 1970] V. M. Kruglov, “Замечание к теории безгранично делимых законов”, Tеоr. Verojatnost. i Primenen 15:2 (1970), 330-336. Translated as "A remark on the theory of infinitely divisible laws" in Theory Prob. Appl. 15:2 (1970), 319-324. MR 42 \#6899 Zbl 0301.60014 
[Lindenstrauss and Tzafriri 1979] J. Lindenstrauss and L. Tzafriri, Classical Banach spaces, II: Function spaces, Ergebnisse der Mathematik und ihrer Grenzgebiete 97, Springer, Berlin, 1979. MR 81c:46001 Zbl 0403.46022

[Marcinkiewicz and Zygmund 1937] J. Marcinkiewicz and A. Zygmund, "Sur les fonctions indépendantes", Fundam. Math. 29 (1937), 60-90. Zbl 0016.40901

[Marcinkiewicz and Zygmund 1938] J. Marcinkiewicz and A. Zygmund, "Quelques théorèmes sur les fonctions indépendantes”, Stud. Math. 7 (1938), 104-120. Zbl 0018.07504

[Prokhorov 1959] Y. V. Prokhorov, "An extremal problem in probability theory", Teor. Verojatnost. $i$ Primenen 4:2 (1959), 211-214. In Russian; translated in Theory Prob. Appl. 4:2 (1959), 201-203. MR 22 \#12587 Zbl 0093.15102

[Rosenthal 1970] H. P. Rosenthal, "On the subspaces of $L^{p}(p>2)$ spanned by sequences of independent random variables", Israel J. Math. 8 (1970), 273-303. MR 42 \#6602 Zbl 0213.19303

Received December 9, 2012.

SERGEY ASTASHKIN

SAMARA STATE UNIVERSITY

PAVLOVA 1

SAMARA

443011

RUSSIA

astashkn@ssu.samara.ru

Fedor A. Sukochev

SCHOOL OF MATHEMATICS AND STATISTICS

UNIVERSITY OF NEW SOUTH WALES

SYDNEY NSW 2052

AUstralia

f.sukochev@unsw.edu.au

DMITRIY ZANIN

SCHOOL OF MATHEMATICS AND STATISTICS

UNIVERSITY OF NEW SOUTH WALES

SYDNEY NSW 2052

AUSTRALIA

d.zanin@unsw.edu.au 


\title{
PACIFIC JOURNAL OF MATHEMATICS
}

\author{
msp.org/pjm
}

Founded in 1951 by E. F. Beckenbach (1906-1982) and F. Wolf (1904-1989)

\section{EDITORS}

Don Blasius (Managing Editor)

Department of Mathematics

University of California

Los Angeles, CA 90095-1555

blasius@math.ucla.edu

\author{
Paul Balmer \\ Department of Mathematics \\ University of California \\ Los Angeles, CA 90095-1555 \\ balmer@math.ucla.edu \\ Robert Finn \\ Department of Mathematics \\ Stanford University \\ Stanford, CA 94305-2125 \\ finn@math.stanford.edu \\ Sorin Popa \\ Department of Mathematics \\ University of California \\ Los Angeles, CA 90095-1555 \\ popa@math.ucla.edu
}

\author{
Vyjayanthi Chari \\ Department of Mathematics \\ University of California \\ Riverside, CA 92521-0135 \\ chari@math.ucr.edu \\ Kefeng Liu \\ Department of Mathematics \\ University of California \\ Los Angeles, CA 90095-1555 \\ liu@math.ucla.edu \\ Jie Qing \\ Department of Mathematics \\ University of California \\ Santa Cruz, CA 95064 \\ qing@ cats.ucsc.edu
}

\section{PRODUCTION}

Silvio Levy, Scientific Editor, production@msp.org

\section{SUPPORTING INSTITUTIONS}

ACADEMIA SINICA, TAIPEI

CALIFORNIA INST. OF TECHNOLOGY

INST. DE MATEMÁTICA PURA E APLICADA

KEIO UNIVERSITY

MATH. SCIENCES RESEARCH INSTITUTE

NEW MEXICO STATE UNIV.

OREGON STATE UNIV.

\author{
STANFORD UNIVERSITY \\ UNIV. OF BRITISH COLUMBIA \\ UNIV. OF CALIFORNIA, BERKELEY \\ UNIV. OF CALIFORNIA, DAVIS \\ UNIV. OF CALIFORNIA, LOS ANGELES \\ UNIV. OF CALIFORNIA, RIVERSIDE \\ UNIV. OF CALIFORNIA, SAN DIEGO \\ UNIV. OF CALIF., SANTA BARBARA
}

\author{
Daryl Cooper \\ Department of Mathematics \\ University of California \\ Santa Barbara, CA 93106-3080 \\ cooper@math.ucsb.edu \\ Jiang-Hua Lu \\ Department of Mathematics \\ The University of Hong Kong \\ Pokfulam Rd., Hong Kong \\ jhlu@maths.hku.hk \\ Paul Yang \\ Department of Mathematics \\ Princeton University \\ Princeton NJ 08544-1000 \\ yang@math.princeton.edu
}

These supporting institutions contribute to the cost of publication of this Journal, but they are not owners or publishers and have no responsibility for its contents or policies.

See inside back cover or msp.org/pjm for submission instructions.

The subscription price for 2014 is US $\$ 410 /$ year for the electronic version, and \$535/year for print and electronic.

Subscriptions, requests for back issues and changes of subscribers address should be sent to Pacific Journal of Mathematics, P.O. Box 4163, Berkeley, CA 94704-0163, U.S.A. The Pacific Journal of Mathematics is indexed by Mathematical Reviews, Zentralblatt MATH, PASCAL CNRS Index, Referativnyi Zhurnal, Current Mathematical Publications and Web of Knowledge (Science Citation Index).

The Pacific Journal of Mathematics (ISSN 0030-8730) at the University of California, c/o Department of Mathematics, 798 Evans Hall \#3840, Berkeley, CA 94720-3840, is published twelve times a year. Periodical rate postage paid at Berkeley, CA 94704, and additional mailing offices. POSTMASTER: send address changes to Pacific Journal of Mathematics, P.O. Box 4163, Berkeley, CA 94704-0163.

PJM peer review and production are managed by EditFLOW ${ }^{\circledR}$ from Mathematical Sciences Publishers.

\section{PUBLISHED BY}

\section{mathematical sciences publishers \\ nonprofit scientific publishing}

http://msp.org/

(C) 2014 Mathematical Sciences Publishers 


\section{PACIFIC JOURNAL OF MATHEMATICS}

Volume $270 \quad$ No. $2 \quad$ August 2014

Disjointification inequalities in symmetric quasi-Banach spaces and 257 their applications

SERgey Astashinin, Fedor A. SukocheV and DMitriy

ZANIN

Hamiltonian evolutions of twisted polygons in parabolic manifolds:

287

The Lagrangian Grassmannian

GLORIA MARÍ BEFFA

On Schwarz-Christoffel mappings

MARTIN CHUAQUi and CHRISTIAN POMMERENKE

Vanishing viscosity in the plane for nondecaying velocity and vorticity, II

ELAINE COZZI

Affine quantum Schur algebras and affine Hecke algebras

QIANG FU

On the classification of Killing submersions and their isometries

JosÉ M. MANZANO

Locally Lipschitz contractibility of Alexandrov spaces and its applications

Ayato Mitsuishi and TAKaO Yamaguchi

Sequences of open Riemannian manifolds with boundary

RAQUEL PERALES and CHRISTINA SORMANI

Invariant differential operators on a class of multiplicity-free spaces 